\title{
Antiproliferative activity of Alpinia officinarum extract in the human breast cancer cell line MCF-7
}

\author{
SUNGHO GHIL \\ Department of Life Science, Kyonggi University, Suwon 443-760, Republic of Korea
}

Received October 18, 2012; Accepted January 21, 2013

DOI: $10.3892 / \mathrm{mmr} .2013 .1305$

\begin{abstract}
Alpinia officinarum (A. officinarum), a member of the ginger family, is used in traditional medicine to treat stomach ache, cold and swelling. Previous studies have demonstrated an anticancer effect of the A. officinarum extract and its major components in several cancer cell lines. However, the molecular mechanisms underlying the activity of this extract in breast cancer cells have not been fully elucidated to date. The aim of the present study was to investigate the molecular mechanisms underlying the activity of a methanolic extract of A. officinarum, by examining its effects on the proliferation of the breast cancer cell line MCF-7. Notably, the extract inhibited MCF-7 cell proliferation in a dose- and time-dependent manner. To further elucidate the molecular mechanism, we examined whether the A. officinarum extract affected cell cycle progression in MCF-7 cells. The extract inhibited S-phase cell cycle progression by suppressing the expression levels of S-phase cell cycle regulatory proteins, including E2F1, cyclin-dependent protein kinase 2 and cyclin A. Additionally, nuclear morphology and flow cytometry with Annexin V/ propidium iodide dual staining demonstrated that apoptosis was induced. Western blot analysis using antibodies against apoptosis-related proteins showed that cell death induced by the extract is mediated via caspase- and mitochondrial-dependent pathways. These findings collectively indicate that the A. officinarum extract exerts an antiproliferative activity in MCF-7 breast cancer cells by inducing S-phase cell cycle arrest and apoptosis.
\end{abstract}

\section{Introduction}

Despite significant improvements in the treatment methods for breast cancer over recent years, including surgical operation, chemotherapy and radiotherapy, breast cancer remains the

Correspondence to: Professor Sungho Ghil, Department of Life Science, Kyonggi University, San 94-6, Iui-dong, Yeongtong-gu, Suwon, Gyeonggi-do 443-760, Republic of Korea

E-mail: shghil@kgu.ac.kr

Key words: Alpinia officinarum, apoptosis, breast cancer, cell cycle, proliferation second leading cause of cancer-induced mortality in women in North America (1). To date, several drugs have been isolated from plants, with the aim of controlling breast cancer. For instance, taxanes and vinca alkaloids isolated from yew tree and rosy periwinkle, respectively, are employed as antimitotic and antimicrotubule agents (2,3). Epipodophyllotoxins isolated from the American mayapple plant exert an anticancer activity by inhibiting topoisomerase II (4). Therefore, it is important to identify natural sources possessing tumor suppressor properties and to investigate the molecular mechanisms underlying these effects, in order to develop anticancer drugs.

Alpinia officinarum (A. officinarum), also known as lesser galangal, is a plant of the ginger family which is commonly found in Southeast Asia. The rhizome of A. officinarum is used in traditional medicine for the treatment of stomach ache, cold and swelling (5). Two major compounds, diarylheptanoids and galangin, have been isolated. Previous studies have demonstrated that the A. officinarum extract and its major components exert an anticancer effect in numerous cancer cell lines, including liver, lung, breast and neuroblastoma (5-8). However, the molecular mechanisms underlying the anticancer activity of this extract in the breast cancer cell line MCF-7 have not been elucidated.

The results of the present study showed that a methanolic extract of A. officinarum rhizome significantly reduces MCF-7 cell proliferation in a dose- and time-dependent manner. The effects of the extract on cell cycle progression and apoptosis in MCF-7 cells were also assessed.

\section{Materials and methods}

A. officinarum extract preparation. Rhizomes of A. officinarum were purchased from the Kyungdong oriental medicine market, Seoul, Korea. Materials $(100 \mu \mathrm{g})$ were extracted with $99.8 \%$ methanol (1 liter) for $72 \mathrm{~h}$ at room temperature. Extracts were evaporated to dryness using a rotary evaporator and dissolved in dimethylsulfoxide (DMSO; Sigma-Aldrich, St. Louis, MO, USA).

Cell culture. The MCF-7 human breast cancer cell line was cultivated in Dulbecco's modified Eagle's medium (DMEM) containing $10 \%$ fetal bovine serum (FBS), $100 \mathrm{U} / \mathrm{ml}$ penicillin and $100 \mu \mathrm{g} / \mathrm{ml}$ streptomycin (HyClone Laboratories, Inc., South Logan, UT, USA) at $37^{\circ} \mathrm{C}$ in a humidified atmosphere of $5 \% \mathrm{CO}_{2}$. 
Cell proliferation assay. The effect of the A. officinarum extract on MCF-7 cell proliferation was measured using the 3-(4,5-dimethylthiazol-z-yl)-2,5-diphenyl tetrazolium bromide (MTT) assay, based on the ability of live cells to cleave the tetrazolium ring to a molecule that absorbs at $570 \mathrm{~nm}(9,10)$. Briefly, cells (3x10\%3/well) were seeded onto 96-well microplates and incubated for $24 \mathrm{~h}$. Next, cells were treated with various concentrations of A. officinarum extract $(0-100 \mu \mathrm{g} / \mathrm{ml})$. At different time points (12, 24, 48 and $72 \mathrm{~h}), 10 \mu \mathrm{l}$ MTT solution ( $5 \mathrm{mg} / \mathrm{ml}$; Sigma-Aldrich) was added to each well, followed by further incubation at $37^{\circ} \mathrm{C}$ for $4 \mathrm{~h}$. At the end of the incubation period, $100 \mu \mathrm{l}$ of isopropyl alcohol dissolved in $5 \%$ of $1 \mathrm{M}$ $\mathrm{HCl}$ was added to solubilize formazan crystals. Absorbance was measured using a SpectraMax ${ }^{\circledR}$ Plus384 microplate reader (Molecular Devices, Sunnyvale, CA, USA). All the measurements were performed in quadruplicate. The cells were never exposed to a DMSO concentration $>0.5 \%$.

Cell cycle analysis using flow cytometry. MCF-7 cells ( $2 \times 10^{5}$ cells/dish) were plated on $100-\mathrm{mm}$ tissue culture dishes, and treated with the indicated concentrations of A. officinarum extract for $48 \mathrm{~h}$. Cells were harvested via trypsinization, washed twice with ice-cold phosphate-buffered saline (PBS), and fixed with $70 \%$ ethanol at $-20^{\circ} \mathrm{C}$ for $20 \mathrm{~min}$. Fixed cells were washed with ice-cold PBS and stained with propidium iodide (PI) solution $(50 \mu \mathrm{g} / \mathrm{ml}$ of PI, $10 \mu \mathrm{g} / \mathrm{ml}$ of RNase A and $3.8 \mathrm{mM}$ sodium citrate in PBS) at $4^{\circ} \mathrm{C}$ for $20 \mathrm{~min}$. Cell cycle distribution was assessed using FACSVantage ${ }^{\mathrm{TM}}$ SE (Becton-Dickinson, San Jose, CA, USA). Data from 10,000 cells/sample were collected and analyzed.

Western blot analysis. MCF-7 cells ( $2 \times 10^{5}$ cells/dish) were plated on a $100-\mathrm{mm}$ tissue culture dish, and treated with the indicated concentrations of A. officinarum extract for $48 \mathrm{~h}$. Next, cells were rinsed twice with ice-cold PBS and scraped using lysis buffer (PBS containing $5 \mathrm{mM} \mathrm{MgCl}_{2}, 1 \mathrm{mM}$ EDTA, $0.1 \%$ Triton $\mathrm{X}-100$ and protease inhibitors). Cell lysates were centrifuged at $12,000 \mathrm{rpm}$ for $10 \mathrm{~min}$ at $4^{\circ} \mathrm{C}$. Protein samples $(60 \mu \mathrm{g})$ were mixed with SDS sample buffer, boiled for $5 \mathrm{~min}$ and subjected to 10 and 12\% SDS-PAGE before electrotransfer to PVDF membrane (Westran S; Whatman, Florham Park, NJ, USA). The membrane was blocked with $5 \%$ non-fat dry milk in TBST for $2 \mathrm{~h}$ at room temperature and incubated with antibodies against E2F1, cyclin-dependent protein kinase 2 (cdk2), cyclin A, caspase-7, poly (ADP-ribose) polymerase (PARP), p53, Bcl-2, Bax (Santa Cruz Biotechnology, Inc., Santa Cruz, CA, USA) or tubulin (Upstate Biotechnology, Temecula, CA, USA), followed by incubation with the corresponding secondary antibodies, goat anti-mouse IgG HRP-conjugate or goat anti-rabbit IgG HRP-conjugate (Zymed, Carlsbad, CA, USA). Protein visualization was achieved using an enhanced chemiluminescence detection kit (West-Zol; Intron Biotechnology, Sungnam, Korea).

Analysis of nuclear morphology. The apoptotic effects of the A. officinarum extract on MCF-7 cells were analyzed via nuclear DNA staining. MCF-7 cells were plated on coverslips at a density of $1 \times 10^{3}$ cells/coverslip and treated with $50 \mu \mathrm{g} / \mathrm{ml}$ of A. officinarum extract. After a 48-h incubation, the cells were fixed with $4 \%$ paraformaldehyde for $10 \mathrm{~min}$, washed twice

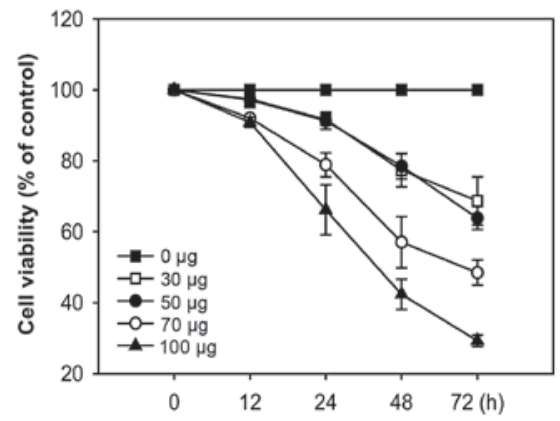

Figure 1. A. officinarum extract exerts an antiproliferative activity in MCF-7 cells. Cells $\left(3 \times 10^{3}\right.$ cells/well) plated on 96 -well microplates were treated with the indicated concentrations of A. officinarum extract for 0-72 $\mathrm{h}$, and proliferation activity was determined using the MTT assay. Data are presented as the means \pm standard error (SE) of values from three independent experiments.

with PBS and stained with $1 \mu \mathrm{g} / \mathrm{ml}$ of Hoechst 33258 (SigmaAldrich) for $20 \mathrm{~min}$. Nuclear morphology was observed under a fluorescence microscope (BX-50; Olympus, Tokyo, Japan).

Annexin V/PI flow cytometric analysis. MCF-7 cells $\left(2 \times 10^{5}\right.$ cells/dish) were plated on a $100-\mathrm{mm}$ tissue culture dish and treated with the indicated amounts of A. officinarum extract for $48 \mathrm{~h}$. The cells were fixed with $70 \%$ ethanol for $20 \mathrm{~min}$, and apoptosis was assessed with an Annexin V FITC Apoptosis Detection kit I (BD Biosciences, Palo Alto, CA, USA), according to the manufacturer's protocol. Flow cytometry analysis was performed using FACSVantage ${ }^{\mathrm{TM}}$ SE. Data from 10,000 cells/sample were collected and analyzed.

\section{Results}

Effects of A. officinarum extract on the proliferation of the human breast cancer cell line MCF-7. To determine whether A. officinarum extract exerts an antiproliferative effect, MCF-7 cells were treated with various concentrations of the extract for the indicated times, and cell proliferation was determined using the MTT-based colorimetric assay. In cells treated with the extract, proliferation was significantly decreased in a time- and dose-dependent manner, clearly demonstrating an antiproliferative effect (Fig. 1).

A. officinarum extract induces $S$-phase cell cycle arrest in MCF-7 cells. Next, we investigated the mechanisms underlying the antiproliferative activity of the extract. MCF-7 cells treated with the indicated amounts of extract for $48 \mathrm{~h}$ were stained with PI and flow cytometric analysis was performed. The A. officinarum extract induced an increase in the proportion of cells in the S-phase in a dose-dependent manner (Fig. 2). Particularly, the cell population in the S-phase was $12.90 \%$ in the untreated control group. After $48 \mathrm{~h}$ of incubation with $100 \mu \mathrm{g} / \mathrm{ml}$ extract, the S-phase population was significantly enhanced to $25.69 \%$ (Fig. 2A).

Western blot analysis was performed to determine the expression levels of S-phase cell cycle regulatory proteins, including E2F1, cdk2 and cyclin A. These proteins are essential for the progression of the S-phase of the cell cycle. The levels of all the proteins examined were significantly suppressed in groups treated with the A. officinarum extract 
A
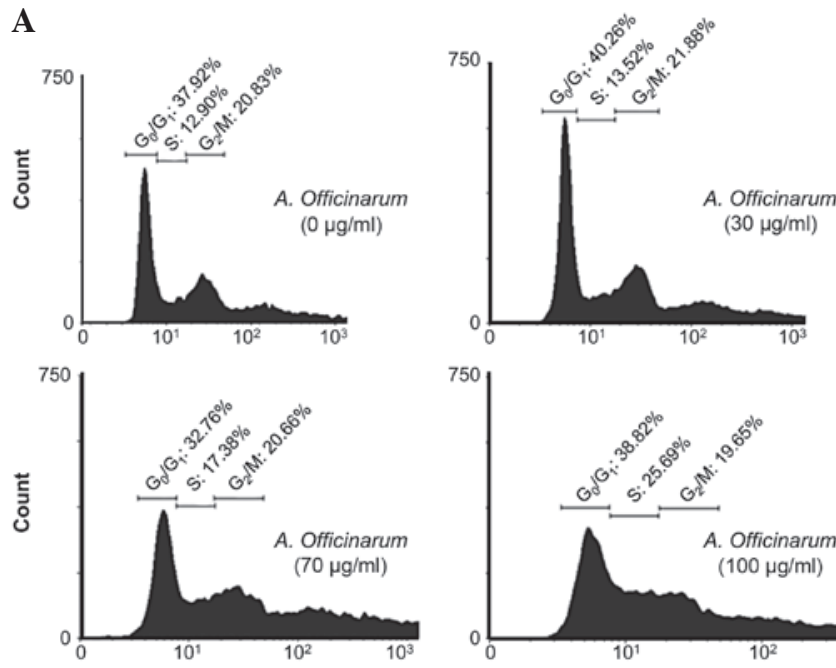

B

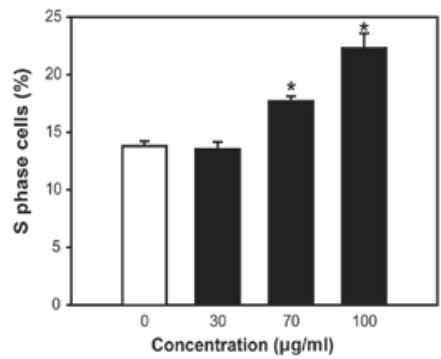

Figure 2. A. officinarum extract induces S-phase cell cycle arrest in MCF-7 cells. Cells $\left(2 \times 10^{5}\right.$ cells/dish) plated on $100-\mathrm{mm}$ dishes were treated with the indicated amounts of extract for $48 \mathrm{~h}$. (A) The cells were stained with PI and subjected to flow cytometry analysis. The proportion of cells in each cell cycle phase is indicated. (B) The percentage of S-phase cells at each extract concentration is shown. Data are presented as the means \pm SE of three independent experiments. ${ }^{*} \mathrm{P}<0.01$, compared with control. PI, propidium iodide.

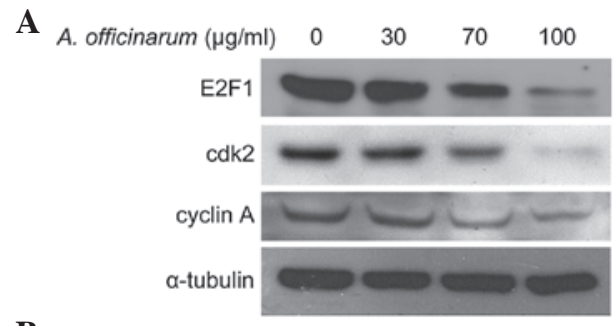

B

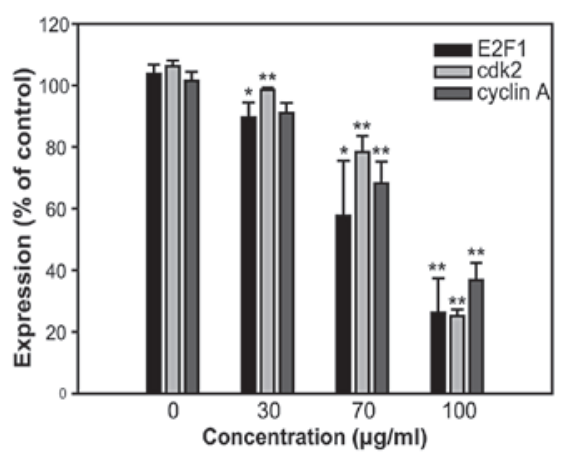

Figure 3. A. officinarum extract inhibits the expression of S-phase regulatory proteins in MCF-7. Cells ( $2 \times 10^{5}$ cells/dish) plated on $100-\mathrm{mm}$ dishes were treated with the indicated concentrations of A. officinarum extract for $48 \mathrm{~h}$. (A) Cell lysates were subjected to western blot analysis using the indicated antibodies. $\alpha$-tubulin was employed as an internal control. (B) Relative band intensities of each protein were quantified using densitometry. Data are presented as the means $\pm \mathrm{SE}$ of three independent experiments. ${ }^{*} \mathrm{P}<0.05$ *** $\mathrm{P}<0.01$, compared with control.
A
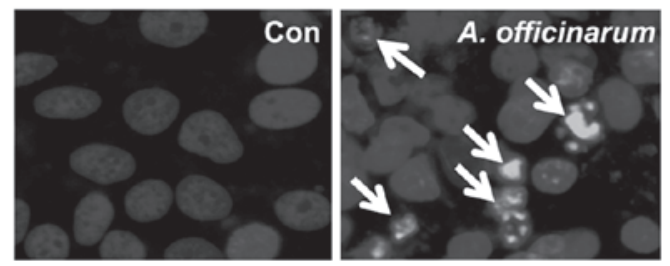

B

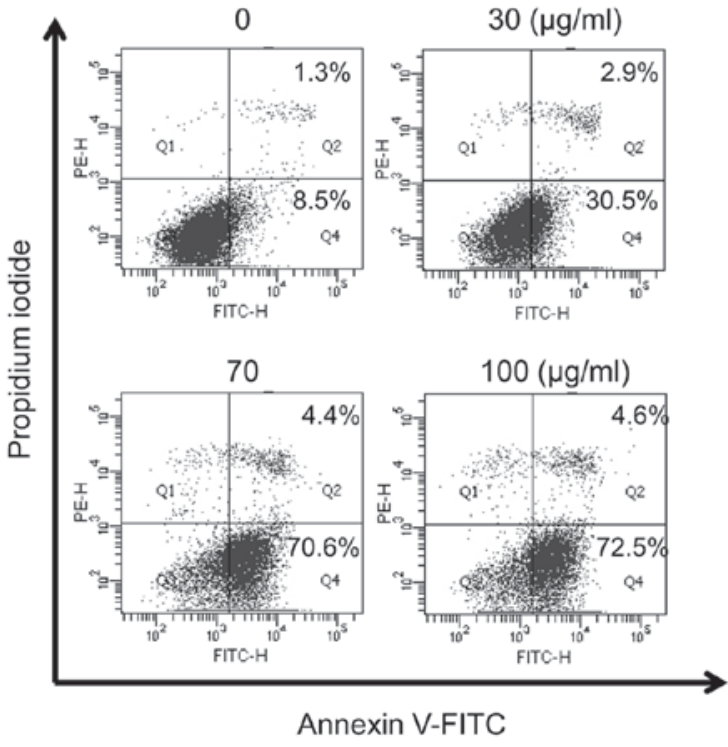

Figure 4. Apoptotic activity of the A. officinarum extract in MCF-7 cells (A) Cells seeded on coverslips $\left(1 \times 10^{3}\right.$ cells/coverslip) were treated with or without $50 \mu \mathrm{g} / \mathrm{ml}$ extract for $48 \mathrm{~h}$ and stained with Hoechst 33258 dye. The arrows indicate nuclear condensation. (B) Cells plated on 100-mm dishes $\left(2 \times 10^{5}\right.$ cells/dish) were treated with the indicated concentrations of extract for $48 \mathrm{~h}$, and subjected to flow cytometric analysis after Annexin V/PI dual staining. The percentage of Annexin $\mathrm{V}^{+} / \mathrm{PI}^{+}$(dead cells) and Annexin $\mathrm{V}^{+} / \mathrm{PI}$ cells (apoptotic cells) is indicated. PI, propidium iodide.

in a dose-dependent manner (Fig. 3). These results suggest that the extract inhibits MCF-7 cell proliferation by inducing S-phase cell cycle arrest.

Apoptotic activity of A. officinarum extract in MCF-7 cells. To further determine whether the extract triggers apoptosis in MCF-7 cells, the cells were treated with or without $50 \mu \mathrm{g}$ extract for $48 \mathrm{~h}$ and stained with Hoechst 33258. Morphological changes in the nucleus were observed under a fluorescence microscope. Nuclear condensation was evident in the presence of A. officinarum extract, while not control cells (Fig. 4A). The apoptosis-promoting potential of the extract was subsequently examined using flow cytometric analysis after Annexin V/ PI dual staining (Fig. 4B). The dot plots show non-apoptotic live cells in the lower left quadrant (Annexin $\mathrm{V}^{-} / \mathrm{PI}^{-}$), apoptotic cells in the lower right quadrant (Annexin $\mathrm{V}^{+} / \mathrm{PI}^{-}$) and dead cells in the upper right quadrant $\left(\right.$ Annexin $\left.\mathrm{V}^{+} / \mathrm{PI}^{+}\right)$. Following treatment with the indicated amounts of $A$. officinarum extract for $48 \mathrm{~h}$, the proportion of apoptotic cells (Annexin $\mathrm{V}^{+} / \mathrm{PI}^{-}$) was significantly increased in a dose-dependent manner. These data collectively indicate that the A. officinarum extract induces apoptosis in MCF-7 cells.

To determine the molecular mechanisms underlying apoptosis induction, cells were treated with the indicated amounts of extract for $48 \mathrm{~h}$, and western blot analysis was 
A

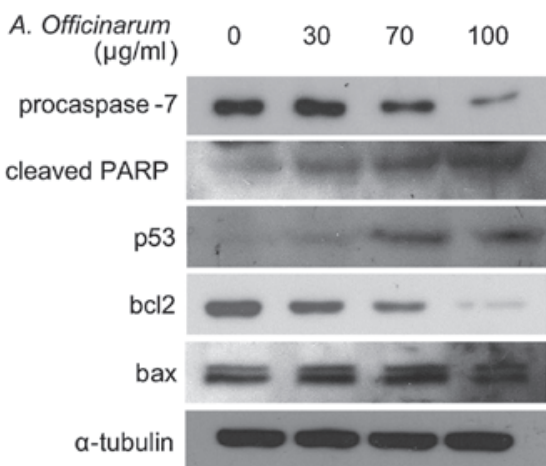

B

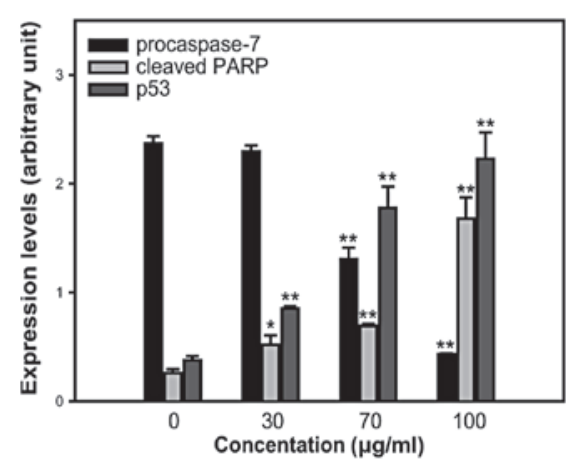

C

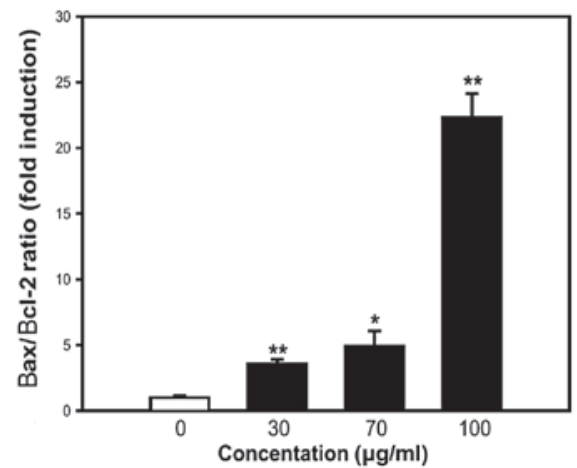

Figure 5. Effects of the A. officinarum extract on the expression levels of apoptosis-related proteins in MCF-7 cells. (A) Cells were treated with the indicated concentrations of extract for $48 \mathrm{~h}$, and subjected to western blot analysis using indicated antibodies. $\alpha$-tubulin was employed as the internal control. (B) Relative band intensities of the indicated proteins were quantified using densitometry. (C) The Bax/Bcl-2 expression ratio is shown. Data are presented as the means $\pm \mathrm{SE}$ of three independent experiments. ${ }^{*} \mathrm{P}<0.05$, ${ }^{* *} \mathrm{P}<0.01$, compared with control.

performed using specific antibodies against apoptosisrelated proteins. The expression level of procaspase-7 was significantly decreased by the extract in a dose-dependent manner (Fig. 5A and B). Procaspase-7, the precursor form of caspase-7, is cleaved into caspase-7. This process is mediated by active caspases or other proteases in response to apoptosis signaling (11). Caspases, a family of cysteine proteases, are the final executors of apoptosis, and are activated via both intrinsic and extrinsic pathways $(12,13)$. Additional evidence of caspase activation was provided by examining the cleavage of the caspase substrate, PARP. Upon introduction of the A. officinarum extract into MCF-7 cells, the presence of an 85-kDa protein fragment, representing the cleaved form of PARP, was increased in a dose-dependent manner (Fig. 5A and $\mathrm{B}$ ), suggesting the induction of caspase-mediated apoptosis in MCF-7 cells.
We also examined the expression of p53, a tumor suppressor, which mediates apoptosis in response to DNA damage. p53 level was significantly increased by the A. officinarum extract in a dose-dependent manner (Fig. 5A and B). Based on the finding that an increase in the $\mathrm{Bax} / \mathrm{Bcl}-2$ ratio indicates mitochondrial dysfunction $(14,15)$, expression levels of Bax and Bcl-2 proteins were further examined. The A. officinarum extract induced a slight increase in Bax and significant decrease in Bcl-2 expression in a dose-dependent manner (Fig. 5A). Subsequent estimation of band intensity further confirmed this dose-dependent increase in the Bax/Bcl-2 ratio (Fig. 5C). These results collectively suggest that apoptosis induced by the A. officinarum extract is mediated via caspaseand mitochondrial-dependent pathways.

\section{Discussion}

In the present study, we investigated the effects of the A. officinarum extract on MCF-7 cell proliferation, and determined the molecular mechanisms underlying its activity. As shown in Fig. 1, the A. officinarum extract inhibited cell proliferation in a dose- and time-dependent manner. Additionally, S-phase cell cycle progression was inhibited in a dose-dependent manner by the extract (Fig. 2). Inhibition of cancer cell proliferation is usually affected by cell cycle arrest. Cell cycle progression is regulated by a complex of cyclin and cdk (16). In particular, the key regulators for $\mathrm{S}$-phase cell cycle progression are cyclin A and cdk2 (17). The cyclin A-cdk2 complex modulates the function of the E2F1 transcription factor, subsequently activating several target genes required for cell cycle progression and DNA synthesis in the S-phase (18). As shown in Fig. 3, the expression levels of cyclin A, cdk2 and E2F1 were downregulated by the A. officinarum extract. Based on these data, it is suggested that the extract suppresses S-phase cell cycle progression by inhibiting regulatory protein expression.

In addition to promoting MCF-7 cell cycle arrest, the A. officinarum extract showed an ability to trigger apoptosis. The cell population displaying nuclear condensation and Annexin $\mathrm{V}^{+} / \mathrm{PI}^{-}$staining was increased following treatment with the extract (Fig. 4). Annexin V binds to phosphatidylserine with high affinity. Under normal conditions, phosphatidylserine is located in the intracellular portion of plasma membrane lipid bilayer. During early apoptosis, phosphatidylserines are redistributed from the intracellular to extracellular portion of the lipid bilayer $(19,20)$. Thus, Annexin $\mathrm{V}^{+}$cells are representative of progressive apoptosis. However, dead cells are also stained with Annexin V, owing to disruption of the cell membrane. In contrast to dead cells, those undergoing progressive apoptosis are undetectable by staining with PI. Dead cells are stained with both Annexin V and PI, whereas viable cells cannot be stained with either dye. The A. officinarum extract induced a marked increase in the Annexin $\mathrm{V}^{+} / \mathrm{PI}^{-}$cell population from 8.5 to $72.5 \%$ (Fig. 4B), indicating the progression of apoptosis.

Apoptosis is induced by the death receptor-mediated extrinsic and mitochondrial cytochrome c-mediated intrinsic pathways. In the extrinsic pathway, extracellular signals, including toxins, hormones, growth factors and cytokines, promote the formation of the death-inducing signaling complex, subsequently activating caspases- 8 and -10 . In the intrinsic pathway, intracellular apoptotic signals, including 
heat, radiation, nutrient deprivation and viral infection, trigger the release of cytochrome $\mathrm{c}$ from mitochondria. Released cytochrome c activates caspases- 9 and -2 by forming apoptosomes (11). Caspases are broadly classified into two groups; initiator (caspase-2, -8, -9 and -10) and executioner (caspases-3, -6 and -7 ) caspases. Executioner caspases are stimulated by active initiator caspases through proteolytic cleavage. Active executioner caspases subsequently cleave various intracellular substrates, including PARP, to perform the cell death program (11). As shown in Fig. 5A and B, the A. officinarum extract induced a dose-dependent decrease and increase in the expression levels of procaspase-7 and cleaved PARP, respectively, supporting a role in the promotion of caspase-mediated apoptosis in MCF-7 cells.

The levels of $\mathrm{p} 53$ and the $\mathrm{Bax} / \mathrm{Bcl}-2$ expression ratio are important biochemical markers of apoptosis. p53 mediates either cell cycle arrest or apoptosis in response to DNA damage, and prevents the replication of damaged DNA via apoptosis $(12,21)$. During apoptosis, p53 induces the transcriptional repression of $\mathrm{Bcl}-2$ and the activation of Bax (22). Therefore, p53 level and $\mathrm{Bax} / \mathrm{Bcl}-2$ ratio are generally increased during apoptosis. Furthermore, the increase in Bax/ Bcl-2 ratio mediates mitochondria-dependent apoptosis by inducing cytochrome $\mathrm{c}$ release from the mitochondria into the cytosol (23). In our experiments, both expression levels of p53 and the Bax/Bcl-2 ratio were significantly increased in the presence of A. officinarum extract in a dose-dependent manner, suggesting an induction of mitochondrial-dependent apoptosis (Fig. 5).

Recently, several compounds have been isolated from A. officinarum extract, including diarylheptanoids and galangin. These compounds exert anticancer effects on numerous cancer cell lines (5-8), and may be responsible for the antiproliferative activity of the extract in MCF-7 cells. Thus, further studies focusing on the molecular mechanisms underlying the anticancer activities of these compounds are essential. Following confirmation of these findings in vivo, the extract or its isolates may be effectively employed to mediate chemotherapeutic and cytostatic activities in human breast cancer.

\section{Acknowledgements}

This study was supported by Kyonggi University Research Grant 2010.

\section{References}

1. DeSantis C, Siegel R, Bandi P and Jemal A: Breast cancer statistics. CA Cancer J Clin 61: 409-418, 2011.

2. Tsavaris N, Kosmas C, Vadiaka M, Kanelopoulos P and Boulamatsis D: Immune changes in patients with advanced breast cancer undergoing chemotherapy with taxanes. Br J Cancer 87: 21-27, 2002.

3. Jordan MA, Horwitz SB, Lobert S and Correia JJ: Exploring the mechanisms of action of the novel microtubule inhibitor vinflunine. Semin Oncol 35: S6-S12, 2008.
4. Baldwin EL and Osheroff N: Etoposide, topoisomerase II and cancer. Curr Med Chem Anticancer Agents 5: 363-372, 2005.

5. An N, Zou ZM, Tian Z, Luo XZ, Yang SL and Xu LZ: Diarylheptanoids from the rhizomes of Alpinia officinarum and their anticancer activity. Fitoterapia 79: 27-31, 2008.

6. Lee CC and Houghton P: Cytotoxicity of plants from Malaysia and Thailand used traditionally to treat cancer. J Ethnopharmacol 100: 237-243, 2005

7. Tabata K, Yamazaki Y, Okada M, Fukumura K, Shimada A, Sun Y, Yasukawa K and Suzuki T: Diarylheptanoids derived from Alpinia officinarum induce apoptosis, S-phase arrest and differentiation in human neuroblastoma cells. Anticancer Res 29: 4981-4988, 2009.

8. Zhang HT, Wu J, Wen M, Su LJ and Luo H: Galangin induces apoptosis in hepatocellular carcinoma cells through the caspase 8/t-Bid mitochondrial pathway. J Asian Nat Prod Res 14: 626-633, 2012.

9. Kim DY, Kang SH and Ghil SH: Cirsium japonicum extract induces apoptosis and anti-proliferation in the human breast cancer cell line MCF-7. Mol Med Rep 3: 427-432, 2010.

10. Jung HW and Ghil SH: A Torilis japonica extract exerts anti-proliferative activities on the U87MG human glioblastoma cell line. Mol Med Rep 3: 1041-1045, 2010.

11. Boatright KM and Salvesen GS: Mechanisms of caspase activation. Curr Opin Cell Biol 15: 725-731, 2003.

12. Das A, Tang KS, Gopalakrishnan S, Waring MJ and Tomasz M: Reactivity of guanine at $\mathrm{m} 5 \mathrm{CpG}$ steps in DNA: evidence for electronic effects transmitted through the base pairs. Chem Biol 6: 461-471, 1999.

13. Budihardjo I, Oliver H,Lutter M, Luo X and Wang X: Biochemical pathways of caspase activation during apoptosis. Annu Rev Cell Dev Biol 15: 269-290, 1999.

14. Yang HL, Chen CS, Chang WH, Lu FJ, Lai YC, Chen CC, Hseu TH, Kuo CT and Hseu YC: Growth inhibition and induction of apoptosis in MCF-7 breast cancer cells by Antrodia camphorata. Cancer Lett 231: 215-227, 2006.

15. Shim HY, Park JH, Paik HD, Nah SY, Kim DS and Han YS: Acacetin-induced apoptosis of human breast cancer MCF-7 cells involves caspase cascade, mitochondria-mediated death signaling and SAPK/JNK1/2-c-Jun activation. Mol Cells 24: 95-104, 2007.

16. Chibazakura T, McGrew SG, Cooper JA, Yoshikawa H and Roberts JM: Regulation of cyclin-dependent kinase activity during mitotic exit and maintenance of genome stability by $\mathrm{p} 21$, p27, and p107. Proc Natl Acad Sci USA 101: 4465-4470, 2004.

17. Chen T and Wong YS: Selenocystine induces S-phase arrest and apoptosis in human breast adenocarcinoma MCF-7 cells by modulating ERK and Akt phosphorylation. J Agric Food Chem 56: 10574-10581, 2008.

18. Xu M, Sheppard KA, Peng CY, Yee AS and Piwnica-Worms H: Cyclin A/CDK2 binds directly to E2F-1 and inhibits the DNA-binding activity of E2F-1/DP-1 by phosphorylation. Mol Cell Biol 14: 8420-8431, 1994.

19. Koopman G, Reutelingsperger CP, Kuijten GA, Keehnen RM, Pals ST and van Oers MH: Annexin V for flow cytometric detection of phosphatidylserine expression on $\mathrm{B}$ cells undergoing apoptosis. Blood 84: 1415-1420, 1994.

20. Martin SJ, Reutelingsperger CP, McGahon AJ, Rader JA, van Schie RC, LaFace DM and Green DR: Early redistribution of plasma membrane phosphatidylserine is a general feature of apoptosis regardless of the initiating stimulus: inhibition by overexpression of Bcl-2 and Abl. J Exp Med 182: 1545-1556, 1995.

21. Zhang XP, Liu F and Wang W: Two-phase dynamics of p53 in the DNA damage response. Proc Natl Acad Sci USA 108: 8990-8995, 2011.

22. Basu A and Haldar S: The relationship between BcI2, Bax and p53: consequences for cell cycle progression and cell death. Mol Hum Reprod 4: 1099-1109, 1998.

23. Kim R, Emi M and Tanabe K: Caspase-dependent and -independent cell death pathways after DNA damage. Oncol Rep 14: 595-599, 2005. 